\title{
LETTER TO THE EDITOR Search for familial clustering of cancer in monoclonal gammopathy of unknown significance
}

Blood Cancer Journal (2016) 6, e445; doi:10.1038/bcj.2016.58; published online 8 July 2016

Monoclonal gammopathy of undetermined significance (MGUS) is an asymptomatic pre-malignant clonal condition which is diagnosed, often coincidentally, through high levels of immunoglobulin derived serum $\mathrm{M}$-protein produced by a plasma cell clone. ${ }^{1,2}$ The prevalence of MGUS increases with age and it is some $3 \%$ at age 60 years and reaching close to $10 \%$ at age over 80 years. ${ }^{3}$ MGUS is a precursor condition for multiple myeloma (MM) and lymphoplasmacytic lymphoma/Waldenström macroglobulinema (LPL/WM). Both MGUS and MM show familial clustering among and between these diseases, suggesting that they share genetic susceptibility. ${ }^{4,5}$ In addition, relatives of MGUS patients have an increased risk of other malignancies of the B-cell lineages, LPL/WM and chronic lymphocytic leukemia (CLL). ${ }^{5}$ No increases were observed in other B-cell or other hematological neoplasms. However, borderline increases of bladder cancers (relative risk 1.4) and spinal cord tumors (3.0) were reported. ${ }^{6}$ Many of these studies originated from Sweden where 4458 persons with MGUS were identified from 1966 to $2005 .^{5}$

For the largest cancer study yet on MGUS we were able to identify 10567 (probably with around 2000 cases overlapping with the above Swedish study) patients from the Swedish health care records who had no previous or concomitant malignancy. Such exclusion of cases is necessary for an unbiased study because diagnosis or suspicion of any cancer may lead to examinations which may result in diagnosis of MGUS that might have otherwise remained undiagnosed. In order to search for internal confirmation of the results, we carried out separate analyses: MGUS risk by familial cancer and cancer risk by familial MGUS.

MGUS patients were identified from the national Hospital Discharge Register between 1997 and 2012 (2655 persons) and the national Outpatient Registry from 2001 to 2012 (7912 persons). MGUS patients were individually linked to the Swedish Cancer Registry and family members were followed-up for diagnosis of cancer from years 1987 to 2012. Any MGUS patient with a previous or concomitant (within 1 year) malignancy were excluded. Finally, the linked data were organized in families, based on the Multigeneration Register. ${ }^{7}$ The offspring generation was born after 1931. The standard diagnostic criteria for MGUS have been the presence of $M$ protein in serum at $<3 \mathrm{~g} / \mathrm{dl}$, fewer than $10 \%$ plasma cells in the bone marrow and lack of symptoms related to monoclonal gammopathy. ${ }^{8}$

Risks for offspring MGUS were calculated by cancer in a proband (including parents and siblings), or, independently, in reverse order, for offspring cancer by MGUS in a proband. Standardized incidence ratios (SIRs), with 95\% confidence intervals, were calculated as the ratio of observed to expected number of cases; the expected numbers were calculated to offspring lacking family history. ${ }^{9}$ SIRs were adjusted by 5-year-age, gender, period (5 years group), socioeconomic status and residential area. ${ }^{10}$

The study was approved by the Ethical Committee of Lund University.

Of a total of 10567 MGUS patients in two generations, 3640 persons belonged to the offspring generation (maximal age 80 years). Among all MGUS patients, men slightly outnumbered women $(51.6 \%, 53.9 \%$ among offspring). The median diagnostic ages were 74 years for all MGUS and 62 years for offspring. Sites were included in Table 1 when over 30 cases were found for 'First degree relative' or if SIRs were significant. The bottom line 'All' nevertheless includes all cancer sites.

In Table 1, the overall SIR for MGUS was marginally increased to 1.05 among offspring of cancer probands. The only solid cancer with a significant association with MGUS was skin squamous cell cancer in first degree relatives (SIR 1.35). MGUS was associated with MM (1.60). MGUS was also associated with non-Hodgkin lymphoma (1.53); the association was highest with LPL/WM (4.07), followed by diffuse large B-cell lymphoma (1.44). The association of MGUS with leukemia was limited to CLL but did not reach statistical significance $(1.49$, all first degree relatives). Because age of onset strongly modifies the risk of many familial cancers, diagnostic age 50 years was considered as the limit among offspring MGUS diagnosis; the early onset cases included no more than $12 \%$ of all cancer associations. At age below 50 years, the overall MGUS risk was 1.19 and it was increased to 1.82 when a first degree relative was diagnosed with bladder cancer. Early onset MGUS was also associated with unspecified non-Hodgkin lymphoma (3.90). Significant associations of late onset MGUS were found for diffuse large B-cell lymphoma (1.44) and myeloma (1.64).

The analyses in reverse order, that is, risk of cancer in offspring when a relative (proband) was diagnosed with MGUS are shown in Table 2. Among solid cancers in offspring the SIR for prostate cancer was increased (1.12) and that for liver cancer was decreased (0.64). The SIRs for MM and non-Hodgkin lymphoma were at the level of Table 1 . The early onset ( $<50$ years) cases cover $34 \%$ of all cases. Among these, offspring risk was increased for early onset small intestinal (3.03) and urinary bladder cancers (SIR 1.87), and for non-thyroid endocrine tumors (1.62). Late onset prostate cancer was increased to 1.13 when a relative was diagnosed with MGUS. Significant lymphoma and LPL/WM risks were found with late onset tumors.

This kind of exploratory analysis in a rather unstudied area of MGUS and cancer is likely to produce false positive results because of multiple comparisons and few previous independent studies are available. The results of the previous studies were cited in the introductory paragraphs also noting that the present MGUS population shared probably $20 \%$ of the cases with the previous Swedish studies. The novel association of MGUS with diffuse large B-cell lymphoma was supported by the two kinds of analyses (SIRs 1.44 and 1.60). The associations of early onset MGUS with bladder cancer was also supported by the two analyses (SIRs 1.82 and 1.87). All other novel associations (prostate, squamous cell skin, small intestine and endocrine glands) were based on individual results and need to wait for confirmation in other datasets. The highest risk in first degree relatives of MGUS patients was found for LPL/WM, which was over 4.0, agreeing with the previous Swedish data. ${ }^{5}$ The present association with myeloma (1.60) was lower, for unknown reasons, than the risk of 2.0-3.0 reported in the Swedish and the US study. ${ }^{4,5}$ The present result on CLL did not reach statistical significance.

It is however noteworthy that several significant associations were found among the early onset cases, suggesting that these 
Letter to the Editor

Table 1. SIR of MGUS in offspring whose first degree relatives were diagnosed with cancer

\begin{tabular}{|c|c|c|c|c|c|c|c|c|c|c|c|c|}
\hline $\begin{array}{l}\text { Cancer in proband } \\
\text { Upper aerodigestive tract }\end{array}$ & $\frac{O}{51}$ & $\begin{array}{c}S I R \\
0.87\end{array}$ & \multicolumn{2}{|c|}{$95 \% \mathrm{Cl}$} & $\frac{0}{6}$ & $\begin{array}{c}\text { SIR } \\
1.06\end{array}$ & \multicolumn{2}{|c|}{$95 \% \mathrm{Cl}$} & $\frac{O}{45}$ & $\begin{array}{c}S I R \\
0.85\end{array}$ & \multicolumn{2}{|c|}{$95 \% \mathrm{Cl}$} \\
\hline Stomach & 89 & 0.86 & 0.69 & 1.06 & 5 & 0.82 & 0.26 & 1.92 & 84 & 0.86 & 0.69 & 1.07 \\
\hline Small intestine & 14 & 1.34 & 0.73 & 2.25 & 3 & 2.60 & 0.49 & 7.69 & 11 & 1.18 & 0.59 & 2.12 \\
\hline Colon & 199 & 1.06 & 0.92 & 1.22 & 15 & 0.84 & 0.47 & 1.39 & 184 & 1.09 & 0.93 & 1.26 \\
\hline Pancreas & 72 & 1.14 & 0.89 & 1.43 & 6 & 1.15 & 0.41 & 2.51 & 66 & 1.14 & 0.88 & 1.45 \\
\hline Lung & 162 & 1.03 & 0.88 & 1.20 & 19 & 1.09 & 0.66 & 1.71 & 143 & 1.02 & 0.86 & 1.20 \\
\hline Breast & 291 & 1.10 & 0.98 & 1.24 & 40 & 1.31 & 0.93 & 1.78 & 251 & 1.07 & 0.95 & 1.22 \\
\hline Cervix & 170 & 1.03 & 0.88 & 1.20 & 33 & 1.07 & 0.73 & 1.50 & 137 & 1.02 & 0.86 & 1.21 \\
\hline Endometrium & 40 & 0.78 & 0.56 & 1.07 & 4 & 0.76 & 0.20 & 1.98 & 36 & 0.79 & 0.55 & 1.09 \\
\hline Ovary & 38 & 0.90 & 0.64 & 1.23 & 4 & 0.96 & 0.25 & 2.47 & 34 & 0.89 & 0.62 & 1.25 \\
\hline Prostate & 297 & 1.06 & 0.94 & 1.19 & 40 & 1.30 & 0.93 & 1.78 & 257 & 1.03 & 0.91 & 1.16 \\
\hline Endocrine glands & 23 & 0.99 & 0.63 & 1.49 & 3 & 1.02 & 0.19 & 3.01 & 20 & 0.99 & 0.60 & 1.53 \\
\hline Non-Hodgkin lymphoma & 66 & 1.53 & 1.18 & 1.95 & 9 & 2.00 & 0.91 & 3.81 & 57 & 1.48 & 1.12 & 1.91 \\
\hline Diffuse large B-cell & 44 & 1.44 & 1.05 & 1.94 & 4 & 1.42 & 0.37 & 3.67 & 40 & 1.44 & 1.03 & 1.97 \\
\hline Lymphoplasmacytic and Waldenstrom & 5 & 4.07 & 1.29 & 9.58 & 1 & 5.59 & 0.00 & 32.02 & 4 & 3.82 & 0.99 & 9.87 \\
\hline Unspecified & 14 & 1.75 & 0.95 & 2.94 & 4 & 3.90 & 1.01 & 10.07 & 10 & 1.43 & 0.68 & 2.64 \\
\hline Myeloma & 32 & 1.60 & 1.09 & 2.26 & 2 & 1.17 & 0.11 & 4.29 & 30 & 1.64 & 1.11 & 2.35 \\
\hline Leukemia & 58 & 1.28 & 0.97 & 1.65 & 7 & 1.44 & 0.57 & 2.98 & 51 & 1.26 & 0.94 & 1.66 \\
\hline Chronic lymphocytic leukemia & 22 & 1.49 & 0.93 & 2.26 & 2 & 1.39 & 0.13 & 5.13 & 20 & 1.50 & 0.91 & 2.32 \\
\hline Cancer of unknown primary & 54 & 1.13 & 0.85 & 1.47 & 6 & 1.33 & 0.48 & 2.92 & 48 & 1.11 & 0.82 & 1.47 \\
\hline All & 2368 & 1.05 & 1.01 & 1.10 & 293 & 1.19 & 1.06 & 1.33 & 2075 & 1.04 & 0.99 & 1.08 \\
\hline
\end{tabular}

Table 2. SIR of cancer in offspring whose first degree relatives were diagnosed with MGUS

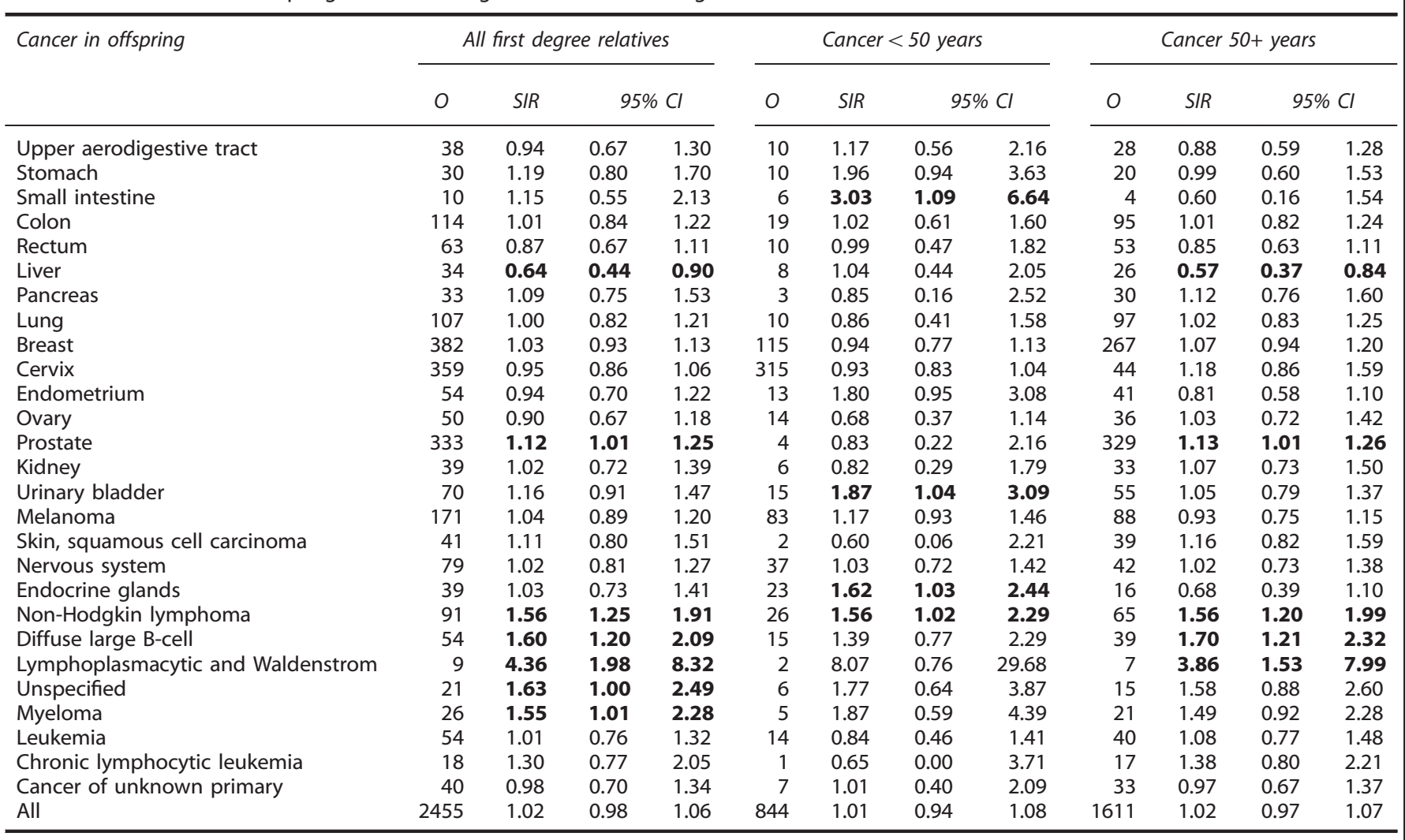

Abbreviations: O, observed number of cases; SIR, standardized incidence ratio; $\mathrm{Cl}$, confidence interval. Bold type: $95 \% \mathrm{Cl}$ does not include 1.00 . 
are more familial than the late onset cases in MGUS as is known for cancers in general. ${ }^{11}$ We reported recently associations of MM with any cancer from the present database. ${ }^{12} \mathrm{MM}$ was found to be associated reliably with colorectal, breast and prostate cancers, non-thyroid endocrine tumors, CLL and cancer of unknown primary. ${ }^{12}$ A single association was also found for MM and bladder cancer. Thus MGUS and MM may share associations with prostate cancers, non-thyroid endocrine tumors and bladder cancer.

In summary, the present large study was able to demonstrate a novel familial association of MGUS with diffuse large B-cell lymphoma and establish the association of early onset MGUS and bladder cancer. These could imply the existence of shared genetic risk factors. Single associations with prostate cancers and nonthyroid endocrine tumors may turn out to be real because these have also been found for MM.

\section{CONFLICT OF INTEREST}

The authors declare no conflict of interest.

\section{ACKNOWLEDGEMENTS}

Supported by Multiple Myeloma Research Foundation, Deutsche Krebshilfe and the Harald Huppert Foundation.

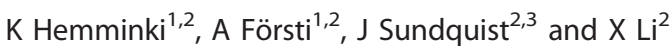
${ }^{1}$ Division of Molecular Genetic Epidemiology, German Cancer Research Center (DKFZ), Heidelberg, Germany;

${ }^{2}$ Center for Primary Health Care Research, Lund University, Malmö, Sweden and

${ }^{3}$ Stanford Prevention Research Center, Stanford University School of Medicine, Stanford, CA, USA E-mail: kari.hemminki@dkfz.de

\section{REFERENCES}

1 Kyle RA, Durie BG, Rajkumar SV, Landgren O, Blade J, Merlini G et al. Monoclonal gammopathy of undetermined significance (MGUS) and smoldering (asymptomatic) multiple myeloma: IMWG consensus perspectives risk factors for progression and guidelines for monitoring and management. Leukemia 2010; 24: 1121-1127.
2 Morgan GJ, Johnson DC, Weinhold N, Goldschmidt H, Landgren O, Lynch HT et al. Inherited genetic susceptibility to multiple myeloma. Leukemia 2014; 28: 518-524.

3 Dispenzieri A, Katzmann JA, Kyle RA, Larson DR, Melton LJ 3rd, Colby CL et al. Prevalence and risk of progression of light-chain monoclonal gammopathy of undetermined significance: a retrospective population-based cohort study. Lancet 2010; 375: 1721-1728.

4 Vachon CM, Kyle RA, Therneau TM, Foreman BJ, Larson DR, Colby CL et al. Increased risk of monoclonal gammopathy in first-degree relatives of patients with multiple myeloma or monoclonal gammopathy of undetermined significance. Blood 2009; 114: 785-790.

5 Landgren O, Kristinsson SY, Goldin LR, Caporaso NE, Blimark C, Mellqvist UH et al. Risk of plasma cell and lymphoproliferative disorders among 14621 first-degree relatives of 4458 patients with monoclonal gammopathy of undetermined significance in Sweden. Blood 2009; 114: 791-795.

6 Kristinsson SY, Goldin LR, Bjorkholm M, Turesson I, Landgren O. Risk of solid tumors and myeloid hematological malignancies among first-degree relatives of patients with monoclonal gammopathy of undetermined significance. Haematologica 2009; 94: 1179-1181.

7 Hemminki K, Ji J, Brandt A, Mousavi SM, Sundquist J. The Swedish FamilyCancer Database 2009: prospects for histology-specific and immigrant studies. Int J Cancer 2010; 126: 2259-2267.

8 Mailankody S, Pfeiffer RM, Kristinsson SY, Korde N, Bjorkholm M, Goldin LR et al. Risk of acute myeloid leukemia and myelodysplastic syndromes after multiple myeloma and its precursor disease (MGUS). Blood 2011; 118: 4086-4092.

9 Breslow NE, Day NE. Statistical methods in cancer research. Volume II--The design and analysis of cohort studies. IARC Sci Publ 1987; 2: 1-406.

10 Esteve J, Benhamou E, Raymond L. Statistical Methods in Cancer Research vol. 128. IARC: Lyon, 1994.

11 Frank C, Fallah M, Sundquist J, Hemminki A, Hemminki K. Population landscape of familial cancer. Sci Rep 2015; 5: 12891

12 Frank C, Fallah M, T. C, Mai EK, Sundquist J, Forsti A et al. Search for familial clustering of multiple myeloma with any cancer. Leukemia 2016; 30: 627-632.

(c) (i) (2) This work is licensed under a Creative Commons Attributioncc. NonCommercial-ShareAlike 4.0 International License. The images or other third party material in this article are included in the article's Creative Commons license, unless indicated otherwise in the credit line; if the material is not included under the Creative Commons license, users will need to obtain permission from the license holder to reproduce the material. To view a copy of this license, visit http:// creativecommons.org/licenses/by-nc-sa/4.0/

(c) The Author(s) 2016 